\title{
Solutions of Boundary Value Problems for Systems of Integro-Differential Equations
}

\author{
Raad N. Butris \& Raveen F. Taher \\ University of Duhok, \\ College of Basic Education, Department of Mathematics, \\ Kurdistan Region, Iraq.
}

\begin{abstract}
In this work, we deals with approximate, uniqueness and stability of a solutions of boundary value problems for new systems of integro-differential equations .We provide a scheme of numerical- analytic method based upon successive approximation for investigate the periodic solution of ordinary differential equations, which are given by Samoilenko. We give sufficient conditions for the solvability of the problem and prove the uniform convergence of the approximations to the limit functions . Also these investigations lead us to the improving and extending the results of Butris.
\end{abstract}

Keywords: Numerical-analytic methods, existence, uniqueness and stability solution, integro-differential equations, boundary value problem.

\section{INTRODUCTION}

Theory of differential equations has been of great interest for many years. It plays an important role in different subjects, such as physics, biology, chemistry, etc. and the study of periodic solutions for non-linear system of differential equations with boundary conditions is very important branch in the differential equation theory $[3,11,14,16,25]$. Many results about the existence and approximation of periodic solutions for system of nonlinear differential equations have been obtained by numerical-analytic methods that were proposed by samoilenko [21,24]. The periodic solutions for some nonlinear systems of integro-differential equations with boundary conditions have been used to study numerous problems for example $[1,2,5,9,10,23,24]$.

The so-called numerical-analytic method for investigating a periodic solution, is widely used for studying solvability of nonlinear boundary value problem and constructing approximate solutions $[4,19,20]$, and it is convenient for finding harmonic oscillations arising in various systems described by ordinary differential equations, differential equations with retarder argument and with impulsive action, integro-differential equations, partial differential equations and differential equations with boundary conditions $[5,6,7,12,13,15,17,18$,$] .$

In [8] Butris and Taher, studied the periodic solution of integro-differential equations depended on special function with singular kernels having the following form

$$
\left.\begin{array}{l}
\frac{d x}{d t}=f\left(t, \gamma(t, \alpha), x(t), \mu, \int_{-\infty}^{t} R(t, \tau)(x(\tau)-y(\tau)) d \tau\right) \\
\frac{d y}{d t}=g\left(t, \gamma(t, \alpha), y(t), \omega, \int_{-\infty}^{t} G(t, \tau)(x(\tau)-y(\tau)) d \tau\right)
\end{array}\right\}
$$

where

$\mu=\int_{a}^{b} \gamma(\tau, \alpha) x(\tau) d \tau$ and $\omega=\int_{c}^{d} \gamma(\tau, \alpha) y(\tau) d \tau, x \in D_{1} \subset R^{n}, y \in D_{2} \subset R^{n}$,

where $D_{1}$ and $D_{2}$ are compact domains where $\gamma(\tau, \alpha)$ is the Gamma function.

Consider the following problem :-

$$
\left.\begin{array}{l}
\frac{d x}{d t}=f\left(t, \beta(t, \alpha), x(t), \mu, \int_{-\infty}^{t} R(t, \tau)(x(\tau)-y(\tau)) d \tau\right) \\
\frac{d y}{d t}=g\left(t, \beta(t, \alpha), y(t), \omega, \int_{-\infty}^{t} G(t, \tau)(x(\tau)-y(\tau)) d \tau\right)
\end{array}\right\}
$$

with boundary conditions

$\left.A_{1} x(\tau)+A_{2} x(\tau+T)=e_{1}\right\}$

$\left.B_{1} y(\tau)+B_{2} y(\tau+T)=e_{2}\right\}$

where

$\mu=\int_{a}^{b} \beta(t, \alpha) x(\tau) d \tau$ and $\omega=\int_{c}^{d} \beta(t, \alpha) y(\tau) d \tau, x \in D_{1} \subset R^{n}, y \in D_{2} \subset R^{n}$,

where $D_{1}$ and $D_{2}$ are compact domains .

Let the vector functions $f(t, \beta(t, \alpha), x, \mu, u)$ and $g(t, \beta(t, \alpha), y, \omega, v)$ are defined and continuous on the domain :-

$\left.(t, \beta(t, \alpha), x, \mu, u) \in R^{1} \times G_{1}=(-\infty, \infty) \times D \times D_{1} \times D_{\mu} \times D_{u}\right)$

$\left.(t, \beta(t, \alpha), y, \omega, v) \in R^{1} \times G_{2}=(-\infty, \infty) \times D \times D_{2} \times D_{\omega} \times D_{v}\right\}$

where $D_{\mu}, D_{\omega}, D_{u}$ and $D_{v}$ are bounded domains subset of Euclidean space $R^{n}$. Also 
$u=\int_{-\infty}^{t} R(t, \tau)(x(\tau)-y(\tau)) d \tau, v=\int_{-\infty}^{t} G(t, \tau)(x(\tau)-y(\tau)) d \tau, D=[\tau, \tau+T] \times(0,1]$. And also $A_{1}=\left(A_{1 i j}\right), A_{2}=$ $\left(A_{2 i j}\right), B_{1}=\left(B_{1 i j}\right)$ and $B_{2}=\left(B_{2 i j}\right)$ are $(n \times n)$ non-negative constant matrices and $e_{1}=\left(e_{11}, e_{12}, e_{13}, \ldots, e_{1 n}\right), e_{2}=$ $\left(e_{21}, e_{22}, e_{23}, \ldots\right.$,

$\left.e_{2 n}\right)$ are positive constant vectors.

Suppose that the functions $f(t, \beta(t, \alpha), x, \mu, u)$ and $g(t, \beta(t, \alpha), y, \omega, v)$ satisfy the following inequalities :

$\left.\|f(t, \beta(t, \alpha), x, \mu, u)\| \leq\|\beta(t, \alpha)\|\|f(t, x, \mu, u)\| \leq M_{\beta} M\right)$

$\left.\|g(t, \beta(t, \alpha), y, \omega, v)\| \leq\|\beta(t, \alpha)\|\|g(t, y, \omega, v)\| \leq N_{\beta} N\right\}$

$\left\|f\left(t, \beta(t, \alpha), x_{1}, \mu_{1}, u_{1}\right)-f\left(t, \beta(t, \alpha), x_{2}, \mu_{2}, u_{2}\right)\right\|$

$\leq M_{\gamma}\left(K_{1}\left\|x_{1}-x_{2}\right\|+K_{2}\left\|\mu_{1}-\mu_{2}\right\|+K_{3}\left\|u_{1}-u_{2}\right\|\right)$

$\left\|g\left(t, \beta(t, \alpha), y_{1}, \omega_{1}, v_{1}\right)-g\left(t, \beta(t, \alpha), y_{2}, \omega_{2}, v_{2}\right)\right\|$

$$
\leq N_{\gamma}\left(L_{1}\left\|y_{1}-y_{2}\right\|+L_{2}\left\|\omega_{1}-\omega_{2}\right\|+L_{3}\left\|v_{1}-v_{2}\right\|\right)
$$

for all $t \in R^{1}, x, x_{1}, x_{2} \in D_{1}, y, y_{1}, y_{2} \in D_{2}, \mu, \omega, u$ and $v$ are belong to $D_{\mu}, D_{\omega}, D_{u}$ and $D_{v}$ respectively, where $M, N, K_{1}, K_{2}, K_{3}, L_{1}, L_{2}$ and $L_{3}$ are positive constants. Also $\beta(t, \alpha)$ is said to be special function( Beta function). The isolated singular kernels $R(t, \tau)$ and $G(t, \tau)$ satisfying the following conditions :-

$\left.\begin{array}{l}\|R(t, \tau)\| \leq h e^{-\alpha(t-\tau)} \\ \|G(t, \tau)\| \leq \sigma e^{-\beta(t-\tau)}\end{array}\right\}$
where $-\infty<0 \leq \tau \leq t \leq \tau+T, \alpha$ and $\delta$ are positive constants.
$\quad$ We defined the non-empty sets as follows :-
$\left.\begin{array}{l}D_{\chi}=G_{1}-\left(\frac{T}{2} M_{\beta} M+B_{1}\right) \\ D_{1 \chi}=G_{2}-\left(\frac{T}{2} N_{\beta} N+B_{2}\right)\end{array}\right\}$

where

$B_{1}=\left\|e_{1} A_{2}^{-1}-\left(A_{1} A_{2}^{-1}+E\right) x_{0}\right\|$ and $B_{2}=\left\|e_{2} B_{2}^{-1}-\left(B_{1} B_{2}^{-1}+E\right) y_{0}\right\|$.

Furthermore, we suppose that the largest eigen-value of the matrix

$\Lambda=\left(\begin{array}{ll}\frac{T}{2} M_{\beta} C_{1} & \frac{T}{2} M_{\beta} C_{2} \\ \frac{T}{2} N_{\beta} C_{3} & \frac{T}{2} N_{\beta} C_{4}\end{array}\right)$ less than one, i.e.

$\lambda_{\text {max }}(\Lambda)=\frac{\varphi_{1}+\sqrt{\varphi_{1}^{2}+4\left(\varphi_{2}-\varphi_{3}\right)}}{2}<1$

where $\varphi_{1}=\frac{T}{2} M_{\beta} C_{1}+\frac{T}{2} N_{\beta} C_{4}, \varphi_{2}=\left(\frac{T}{2} M_{\beta} C_{2}\right)\left(\frac{T}{2} N_{\beta} C_{3}\right)$ and

$\varphi_{3}=\left(\frac{T}{2} M_{\beta} C_{1}\right)\left(\frac{T}{2} N_{\gamma} C_{4}\right)$

Define the sequence of functions on the domain (1.3) by the following

$x_{m+1}\left(t, x_{0}, y_{0}\right)=x_{0}+\int_{\tau}^{t}\left[f\left(\tau, \beta(\tau, \alpha), x_{m}\left(\tau, x_{0}, y_{0}\right), \mu_{m}, u_{m}\right)-\frac{1}{T} \int_{\tau}^{\tau+T} f(\tau, \beta(\tau, \alpha)\right.$

$$
\left.\left., x_{m}\left(\tau, x_{0}, y_{0}\right), \mu_{m}, u_{m}\right)\right] d \tau+\frac{t-\tau}{T}\left[e_{1} A_{2}^{-1}-\left(A_{1} A_{2}^{-1}+E\right) x_{0}\right]
$$

and

$y_{m+1}\left(t, x_{0}, y_{0}\right)=y_{0}+\int_{\tau}^{t}\left[g\left(\tau, \beta(\tau, \alpha), y_{m}\left(\tau, x_{0}, y_{0}\right), \omega_{m}, v_{m}\right)-\frac{1}{T} \int_{\tau}^{\tau+T} g(\tau, \beta(\tau, \alpha)\right.$

$$
\left.\left., y_{m}\left(\tau, x_{0}, y_{0}\right), \omega_{m}, v_{m}\right)\right] d \tau+\frac{t-\tau}{T}\left[e_{2} B_{2}^{-1}-\left(B_{1} B_{2}^{-1}+E\right) y_{0}\right]
$$

with

$x_{0}\left(t, x_{0}, y_{0}\right)=x_{0}, y_{0}\left(t, x_{0}, y_{0}\right)=y_{0}, \quad m=0,1,2, \ldots$.

where

$\mu_{m}=\int_{a}^{b} \beta(\tau, \alpha) x_{m}\left(\tau, x_{0}, y_{0}\right) d \tau, \omega_{m}=\int_{c}^{d} \beta(\tau, \alpha) y_{m}\left(\tau, x_{0}, y_{0}\right) d \tau$

$u_{m}=\int_{-\infty}^{t} R(t, \tau)\left(x_{m}\left(\tau, x_{0}, y_{0}\right)-y_{m}\left(\tau, x_{0}, y_{0}\right)\right) d \tau$ 
$v_{m}=\int_{-\infty}^{t} G(t, \tau)\left(x_{m}\left(\tau, x_{0}, y_{0}\right)-y_{m}\left(\tau, x_{0}, y_{0}\right)\right) d \tau$

Lemma 1.1.[19] Let $f(t, \gamma(t, \alpha), x, \mu, u)$ and $g(t, \gamma(t, \alpha), y, \omega, v)$ be vectors which are defined on the interval [ $\tau, \tau+T]$, then $\left(\begin{array}{l}\left\|Q_{1}\left(t, x_{0}, y_{0}\right)\right\| \\ \left\|Q_{2}\left(t, x_{0}, y_{0}\right)\right\|\end{array}\right) \leq\left(\begin{array}{c}\alpha(t) M_{\beta} M+B_{1} \\ \alpha(t) N_{\beta} N+B_{2}\end{array}\right)$ satisfies for $\tau \leq t \leq \tau+T$ and $\alpha(t) \leq \frac{T}{2}$

where $\alpha(t)=2(t-\tau)\left(1-\frac{t-\tau}{T}\right)$ for all $t \in[\tau, \tau+T]$,

$Q_{1}\left(t, x_{0}, y_{0}\right)=\int_{\tau}^{t}\left[f\left(\tau, \beta(\tau, \alpha), x\left(\tau, x_{0}, y_{0}\right), \mu, u\right)-\frac{1}{T} \int_{\tau}^{\tau+T} f\left(\tau, \beta(\tau, \alpha), x\left(\tau, x_{0}, y_{0}\right)\right.\right.$,

$\mu, u) d \tau] d \tau+\frac{t-\tau}{T}\left[e_{1} A_{2}^{-1}-\left(A_{1} A_{2}^{-1}+E\right) x_{0}\right]$

$Q_{2}\left(t, x_{0}, y_{0}\right)=\int_{\tau}^{t}\left[g\left(\tau, \beta(\tau, \alpha), y\left(\tau, x_{0}, y_{0}\right), \omega, v\right)-\frac{1}{T} \int_{\tau}^{\tau+T} g\left(\tau, \beta(\tau, \alpha), y\left(\tau, x_{0}, y_{0}\right)\right.\right.$,

$$
\omega, v) d \tau] d \tau+\frac{t-\tau}{T}\left[e_{2} B_{2}^{-1}-\left(B_{1} B_{2}^{-1}+E\right) y_{0}\right]
$$

Proof .

$$
\begin{aligned}
& \left\|Q_{1}\left(t, x_{0}, y_{0}\right)\right\| \leq\left(1-\frac{t-\tau}{T}\right) \int_{\tau}^{t}\|\beta(\tau, \alpha)\|\left\|f\left(\tau, x\left(\tau, x_{0}, y_{0}\right), \mu, u\right)\right\| d \tau \\
& +\frac{t-\tau}{T} \int_{t}^{\tau+T}\|\beta(\tau, \alpha)\|\left\|f\left(\tau, x\left(\tau, x_{0}, y_{0}\right), \mu, u\right)\right\| d \tau+\left\|e_{1} A_{2}^{-1}-\left(A_{1} A_{2}^{-1}+E\right) x_{0}\right\| \\
& \leq \alpha(t) M_{\beta} M+B_{1}
\end{aligned}
$$

so that

$\left\|Q_{1}\left(t, x_{0}, y_{0}\right)\right\| \leq \alpha(t) M_{\beta} M+B_{1}$

and

$\left\|Q_{2}\left(t, x_{0}, y_{0}\right)\right\| \leq \alpha(t) N_{\beta} N+B_{2}$

From (1.13) and (1.14) we conclude that the inequality (1.12) holds .

\section{APPROXIMATION SOLUTION FOR THE SYSTEM (1.1) WITH BOUNDARY CONDITIONS (1.2).}

In this section, we shall the investigation of approximation solution of the system (1.1) with boundary conditions (1.2) by the following :-

Theorem 2.1: If the system (1.1) with boundary conditions (1.2) defined in the domain (1.3), continuous in $t, x, y$ and satisfy the inequalities (1.4) to (1.7) and the conditions (1.8) and (1.9). Then the sequence of functions (1.10) and (1.11) convergent uniformly as $m \rightarrow \infty$ on the domain :-

$\left(t, x_{0}, y_{0}\right) \in[\tau, \tau+T] \times D_{\chi} \times D_{1 \chi}$

to the limit function $\left(\begin{array}{c}x\left(t, x_{0}, y_{0}\right) \\ y\left(t, x_{0}, y_{0}\right)\end{array}\right)$ defined on the domain (2.1) which is periodic in $t$ of period $T$ and satisfying the following integral equations :-

$x\left(t, x_{0}, y_{0}\right)=x_{0}+\int_{\tau}^{t}\left[f\left(\tau, \beta(\tau, \alpha), x\left(\tau, x_{0}, y_{0}\right), \mu, u\right)-\frac{1}{T} \int_{\tau}^{\tau+T} f(\tau, \beta(\tau, \alpha)\right.$,

$$
\left.\left.x\left(\tau, x_{0}, y_{0}\right), \mu, u\right)\right] d \tau+\frac{t-\tau}{T}\left[e_{1} A_{2}^{-1}-\left(A_{1} A_{2}^{-1}+E\right) x_{0}\right]
$$

and

$y\left(t, x_{0}, y_{0}\right)=y_{0}+\int_{\tau}^{t}\left[g\left(\tau, \beta(\tau, \alpha), y\left(\tau, x_{0}, y_{0}\right), \omega, v\right)-\frac{1}{T} \int_{\tau}^{\tau+T} g(\tau, \beta(\tau, \alpha)\right.$, 


$$
\left.\left.y\left(\tau, x_{0}, y_{0}\right), \omega, v\right)\right] d \tau+\frac{t-\tau}{T}\left[e_{2} B_{2}^{-1}-\left(B_{1} B_{2}^{-1}+E\right) y_{0}\right]
$$

which are unique solutions on the domain (2.1), provided that

$$
\left(\begin{array}{l}
\left\|x\left(t, x_{0}, y_{0}\right)-x_{0}\right\| \\
\left\|y\left(t, x_{0}, y_{0}\right)-y_{0}\right\|
\end{array}\right) \leq\left(\begin{array}{l}
\frac{T}{2} M_{\beta} M+B_{1} \\
\frac{T}{2} N_{\beta} N+B_{2}
\end{array}\right)
$$

and

$$
\left(\begin{array}{l}
\left\|x\left(t, x_{0}, y_{0}\right)-x_{m}\left(t, x_{0}, y_{0}\right)\right\| \\
\left\|y\left(t, x_{0}, y_{0}\right)-y_{m}\left(t, x_{0}, y_{0}\right)\right\|
\end{array}\right) \leq \Lambda^{m}(E-\Lambda)^{-1} \eta
$$

for all $m \geq 0, x_{0} \in D_{\chi}, y_{0} \in D_{1 \chi}$ and $t \in R^{1}$, where $E$ is identity matrix.

Proof. Setting $m=0$ in the sequence of functions (1.10),(1.11) and using Lemma 1.1, we have

$$
\begin{gathered}
\left\|x_{1}\left(t, x_{0}, y_{0}\right)-x_{0}\right\|=\| \int_{\tau}^{t}\left[f \left(\tau, \beta(\tau, \alpha), x_{0}, \int_{a}^{b} \gamma(\tau, \alpha) x_{0} d \tau, \int_{-\infty}^{t} R(t, \tau)\right.\right. \\
\left.\left(x_{0}-y_{0}\right) d \tau\right)-\frac{1}{T} \int_{\tau}^{\tau+T} f\left(\tau, \beta(\tau, \alpha), x_{0}, \int_{a}^{b} \gamma(\tau, \alpha) x_{0} d \tau, \int_{-\infty}^{t} R(t, \tau)\right. \\
\left.\left(x_{0}-y_{0}\right) d \tau\right)+\frac{t-\tau}{T}\left[e_{1} A_{2}^{-1}-\left(A_{1} A_{2}^{-1}+E\right) x_{0}\right] \|
\end{gathered}
$$

Hence

$\left\|x_{1}\left(t, x_{0}, y_{0}\right)-x_{0}\right\| \leq \alpha(t) M_{\beta} M+B_{1} \leq \frac{T}{2} M_{\beta} M+B_{1}$

for all $x_{1}\left(t, x_{0}, y_{0}\right) \in G_{1}$, for all $t \in[\tau, \tau+T], x_{0} \in D_{\chi}$.

and similarly, we get

$\left\|y_{1}\left(t, x_{0}, y_{0}\right)-y_{0}\right\| \leq \alpha(t) N_{\beta} N+B_{2} \leq \frac{T}{2} N_{\beta} N+B_{2}$

for all $y_{1}\left(t, x_{0}, y_{0}\right) \in G_{2}$, for all $t \in[\tau, \tau+T], y_{0} \in D_{1 \chi}$.

Thus, by mathematical induction, we can prove that

$$
\left.\begin{array}{c}
\left\|x_{m}\left(t, x_{0}, y_{0}\right)-x_{0}\right\| \leq \frac{T}{2} M_{\beta} M+B_{1} \\
\left\|y_{m}\left(t, x_{0}, y_{0}\right)-y_{0}\right\| \leq \frac{T}{2} N_{\beta} N+B_{2}
\end{array}\right\}
$$

i.e. $x_{m}\left(t, x_{0}, y_{0}\right) \in G_{1}, y_{m}\left(t, x_{0}, y_{0}\right) \in G_{2}, x_{0} \in D_{\chi}, y_{0} \in D_{1 \chi}$, for all $t \in[\tau, \tau+T]$,

$m=0,1,2, \ldots$.

Rewrite the inequality (2.8) by the vector from, then we get (2.4).

We claim that the sequence of functions (1.10) and (1.11) are uniformly convergent on the domain (2.1).

We begin by finding an estimate for $\left\|x_{m+1}\left(t, x_{0}, y_{0}\right)-x_{m}\left(t, x_{0}, y_{0}\right)\right\|$ and

$\left\|y_{m+1}\left(t, x_{0}, y_{0}\right)-y_{m}\left(t, x_{0}, y_{0}\right)\right\|$, since

$x_{m+1}\left(t, x_{0}, y_{0}\right)-x_{m}\left(t, x_{0}, y_{0}\right)=\int_{\tau}^{t}\left[f\left(\tau, \beta(\tau, \alpha), x_{m}\left(\tau, x_{0}, y_{0}\right), \mu_{m}, u_{m}\right)\right.$

$$
\left.-f\left(\tau, \beta(\tau, \alpha), x_{m-1}\left(\tau, x_{0}, y_{0}\right), \mu_{m-1}, u_{m-1}\right)\right]-\frac{1}{T} \int_{\tau}^{\tau+T}[f(\tau, \beta(\tau, \alpha),
$$

$\left.\left.\left.x_{m}\left(\tau, x_{0}, y_{0}\right), \mu_{m}, u_{m}\right)-f\left(\tau, \beta(\tau, \alpha), x_{m-1}\left(\tau, x_{0}, y_{0}\right), \mu_{m-1}, u_{m-1}\right)\right] d \tau\right] d \tau$

and

$y_{m+1}\left(t, x_{0}, y_{0}\right)-y_{m}\left(t, x_{0}, y_{0}\right)=\int_{\tau}^{t}\left[g\left(\tau, \beta(\tau, \alpha), y_{m}\left(\tau, x_{0}, y_{0}\right), \omega_{m}, v_{m}\right)\right.$

$$
\left.-g\left(\tau, \beta(\tau, \alpha), y_{m-1}\left(\tau, x_{0}, y_{0}\right), \omega_{m-1}, v_{m-1}\right)\right]-\frac{1}{T} \int_{\tau}^{\tau+T}[g(\tau, \gamma(\tau, \alpha),
$$


$\left.\left.\left.y_{m}\left(\tau, x_{0}, y_{0}\right), \omega_{m}, v_{m}\right)-g\left(\tau, \beta(\tau, \alpha), y_{m-1}\left(\tau, x_{0}, y_{0}\right), \omega_{m-1}, v_{m-1}\right)\right] d \tau\right] d \tau$

where

$$
\begin{gathered}
\mu_{m-1}=\int_{a}^{b} \beta(\tau, \alpha) x_{m-1}\left(\tau, x_{0}, y_{0}\right) d \tau, \omega_{m-1}=\int_{c}^{d} \beta(\tau, \alpha) y_{m-1}\left(\tau, x_{0}, y_{0}\right) d \tau \\
u_{m-1}=\int_{-\infty}^{t} R(t, \tau)\left(x_{m-1}\left(\tau, x_{0}, y_{0}\right)-y_{m-1}\left(\tau, x_{0}, y_{0}\right)\right) d \tau \\
v_{m-1}=\int_{-\infty}^{t} G(t, \tau)\left(x_{m-1}\left(\tau, x_{0}, y_{0}\right)-y_{m-1}\left(\tau, x_{0}, y_{0}\right)\right) d \tau
\end{gathered}
$$

Using the inequalities (1.5) and (1.6), we have

$\left\|x_{m+1}\left(t, x_{0}, y_{0}\right)-x_{m}\left(t, x_{0}, y_{0}\right)\right\| \leq \alpha(t) M_{\beta} C_{1}\left\|x_{m}\left(t, x_{0}, y_{0}\right)-x_{m-1}\left(t, x_{0}, y_{0}\right)\right\|$

$$
+\alpha(t) M_{\beta} C_{2}\left\|y_{m}\left(t, x_{0}, y_{0}\right)-y_{m-1}\left(t, x_{0}, y_{0}\right)\right\|
$$

where $C_{1}=K_{1}+K_{2} M_{\beta}(b-a)+\frac{h}{\alpha} K_{3}$ and $C_{2}=\frac{h}{\alpha} K_{3}$

And

$\left\|y_{m+1}\left(t, x_{0}, y_{0}\right)-y_{m}\left(t, x_{0}, y_{0}\right)\right\| \leq \alpha(t) N_{\beta} C_{3}\left\|x_{m}\left(t, x_{0}, y_{0}\right)-x_{m-1}\left(t, x_{0}, y_{0}\right)\right\|$

$$
+\alpha(t) N_{\beta} C_{4}\left\|y_{m}\left(t, x_{0}, y_{0}\right)-y_{m-1}\left(t, x_{0}, y_{0}\right)\right\|
$$

where $C_{3}=\frac{\sigma}{\beta} L_{3}$ and $C_{4}=L_{1}+L_{2} N_{\beta}(d-c)+\frac{\sigma}{\beta} L_{3}$

Rewrite the inequalities (2.9) and (2.10) in vector form

$\left(\begin{array}{l}\left\|x_{m+1}\left(t, x_{0}, y_{0}\right)-x_{m}\left(t, x_{0}, y_{0}\right)\right\| \\ \left\|y_{m+1}\left(t, x_{0}, y_{0}\right)-y_{m}\left(t, x_{0}, y_{0}\right)\right\|\end{array}\right) \leq \Lambda\left(\begin{array}{l}\left\|x_{m}\left(t, x_{0}, y_{0}\right)-x_{m-1}\left(t, x_{0}, y_{0}\right)\right\| \\ \left\|y_{m}\left(t, x_{0}, y_{0}\right)-y_{m-1}\left(t, x_{0}, y_{0}\right)\right\|\end{array}\right)$

If we set $m=1$ in (1.23) and (1.24), we get

$\left(\begin{array}{l}\left\|x_{2}\left(t, x_{0}, y_{0}\right)-x_{1}\left(t, x_{0}, y_{0}\right)\right\| \\ \left\|y_{2}\left(t, x_{0}, y_{0}\right)-y_{1}\left(t, x_{0}, y_{0}\right)\right\|\end{array}\right) \leq \Lambda\left(\begin{array}{l}\left\|x_{1}\left(t, x_{0}, y_{0}\right)-x_{0}\right\| \\ \left\|y_{1}\left(t, x_{0}, y_{0}\right)-y_{0}\right\|\end{array}\right)$

Next, set $m=2$ and use (2.12), we have

$\left(\begin{array}{l}\left\|x_{3}\left(t, x_{0}, y_{0}\right)-x_{2}\left(t, x_{0}, y_{0}\right)\right\| \\ \left\|y_{3}\left(t, x_{0}, y_{0}\right)-y_{2}\left(t, x_{0}, y_{0}\right)\right\|\end{array}\right) \leq \Lambda^{2}\left(\begin{array}{c}\left\|x_{1}\left(t, x_{0}, y_{0}\right)-x_{0}\right\| \\ \left\|y_{1}\left(t, x_{0}, y_{0}\right)-y_{0}\right\|\end{array}\right)$

Setting $m=2$, yields in the same way

$\left(\begin{array}{l}\left\|x_{4}\left(t, x_{0}, y_{0}\right)-x_{3}\left(t, x_{0}, y_{0}\right)\right\| \\ \left\|y_{4}\left(t, x_{0}, y_{0}\right)-y_{3}\left(t, x_{0}, y_{0}\right)\right\|\end{array}\right) \leq \Lambda^{3}\left(\begin{array}{c}\left\|x_{1}\left(t, x_{0}, y_{0}\right)-x_{0}\right\| \\ \left\|y_{1}\left(t, x_{0}, y_{0}\right)-y_{0}\right\|\end{array}\right)$

and by mathematical induction, we obtain that

$\left(\begin{array}{l}\left\|x_{m+1}\left(t, x_{0}, y_{0}\right)-x_{m}\left(t, x_{0}, y_{0}\right)\right\| \\ \left\|y_{m+1}\left(t, x_{0}, y_{0}\right)-y_{m}\left(t, x_{0}, y_{0}\right)\right\|\end{array}\right) \leq \Lambda^{m}\left(\begin{array}{l}\left\|x_{m}\left(t, x_{0}, y_{0}\right)-x_{m-1}\left(t, x_{0}, y_{0}\right)\right\| \\ \left\|y_{m}\left(t, x_{0}, y_{0}\right)-y_{m-1}\left(t, x_{0}, y_{0}\right)\right\|\end{array}\right)$

Now, from $m=1,2, \ldots$ and $p \geq 1$, we have

$$
\begin{aligned}
& \left(\begin{array}{l}
\left\|x_{m+p}\left(t, x_{0}, y_{0}\right)-x_{m}\left(t, x_{0}, y_{0}\right)\right\| \\
\left\|y_{m+p}\left(t, x_{0}, y_{0}\right)-y_{m}\left(t, x_{0}, y_{0}\right)\right\|
\end{array}\right) \leq\left(\begin{array}{l}
\left\|x_{m+1}\left(t, x_{0}, y_{0}\right)-x_{m}\left(t, x_{0}, y_{0}\right)\right\| \\
\left\|y_{m+1}\left(t, x_{0}, y_{0}\right)-y_{m}\left(t, x_{0}, y_{0}\right)\right\|
\end{array}\right)+\cdots+\left(\begin{array}{l}
\left\|x_{m+p}\left(t, x_{0}, y_{0}\right)-x_{m+p-1}\left(t, x_{0}, y_{0}\right)\right\| \\
\left\|y_{m+p}\left(t, x_{0}, y_{0}\right)-y_{m+p-1}\left(t, x_{0}, y_{0}\right)\right\|
\end{array}\right) \\
& +\left(\begin{array}{c}
\left\|x_{m+2}\left(t, x_{0}, y_{0}\right)-x_{m+1}\left(t, x_{0}, y_{0}\right)\right\| \\
\left\|y_{m+2}\left(t, x_{0}, y_{0}\right)-y_{m+1}\left(t, x_{0}, y_{0}\right)\right\|
\end{array}\right)+\cdots \\
& \leq \Lambda^{m}\left(\begin{array}{c}
\left\|x_{1}\left(t, x_{0}, y_{0}\right)-x_{0}\right\| \\
\left\|y_{1}\left(t, x_{0}, y_{0}\right)-y_{0}\right\|
\end{array}\right)+\Lambda^{m+1}\left(\begin{array}{l}
\left\|x_{1}\left(t, x_{0}, y_{0}\right)-x_{0}\right\| \\
\left\|y_{1}\left(t, x_{0}, y_{0}\right)-y_{0}\right\|
\end{array}\right)+\cdots+ \\
& \Lambda^{m+p-1}\left(\begin{array}{l}
\left\|x_{1}\left(t, x_{0}, y_{0}\right)-x_{0}\right\| \\
\left\|y_{1}\left(t, x_{0}, y_{0}\right)-y_{0}\right\|
\end{array}\right)
\end{aligned}
$$


Therefore,

$\left(\begin{array}{c}\| x_{m+p}\left(t, x_{0}, y_{0}\right)-x_{m}\left(t, x_{0}, y_{0}\right) \\ \left\|p\left(t, x_{0}, y_{0}\right)-y_{m}\left(t, x_{0}, y_{0}\right)\right\|\end{array}\right) \leq \Lambda^{m}\left(E+\Lambda+\cdots+\Lambda^{p-1}\right) \eta$

where $\eta=\left(\begin{array}{l}\frac{T}{2} M_{\beta} M+B_{1} \\ \frac{T}{2} N_{\beta} N+B_{2}\end{array}\right)$

For all $t \in[\tau, \tau+T], x_{0} \in D_{\chi}$ and $y_{0} \in D_{1 \chi}$.

Since $\frac{\varphi_{1}+\sqrt{\varphi_{1}^{2}+4\left(\varphi_{2}-\varphi_{3}\right)}}{2}<1$ and $\lim _{m \rightarrow \infty} \Lambda^{m}=0$, so that the right side of (2.13) tends

to zero. Therefore, the sequence of function $\left(\begin{array}{c}x\left(t, x_{0}, y_{0}\right) \\ y\left(t, x_{0}, y_{0}\right)\end{array}\right)$ is convergent uniformly on the domain (2.1) to the limit function $\left(\begin{array}{c}x\left(t, x_{0}, y_{0}\right) \\ y\left(t, x_{0}, y_{0}\right)\end{array}\right)$ which is defined on the same domain.

Let

$\lim _{m \rightarrow \infty}\left(\begin{array}{c}x_{m}\left(t, x_{0}, y_{0}\right) \\ y_{m}\left(t, x_{0}, y_{0}\right)\end{array}\right)=\left(\begin{array}{c}x\left(t, x_{0}, y_{0}\right) \\ y\left(t, x_{0}, y_{0}\right)\end{array}\right)$

Since the sequence of functions (1.10) and (1.11) are conations in $t$ of period $T$, then the limiting function $\left(\begin{array}{c}x\left(t, x_{0}, y_{0}\right) \\ y\left(t, x_{0}, y_{0}\right)\end{array}\right)$ is also conations. Also, by the Lemma 1.1 and the inequality (2.13) the inequalities (2.4) and (2.5) are holds for all $m \geq 0$.

By using the relation (2.14) and proceeding in (1.10) and (1.11) to limit, when $m \rightarrow 0$, convinces us that the limiting function $\left(\begin{array}{c}x\left(t, x_{0}, y_{0}\right) \\ y\left(t, x_{0}, y_{0}\right)\end{array}\right)$ is the a solution of the integral equations (1.10) and (1.11).

Finally, we must still prove that $\left(\begin{array}{c}x\left(t, x_{0}, y_{0}\right) \\ y\left(t, x_{0}, y_{0}\right)\end{array}\right)$ is a unique solution of (1.1)

with boundary conditions (1.2). Assume that $\left(\begin{array}{l}\hat{x} \\ \hat{y}\end{array}\right)$ is another solution for the system (1.1) with boundary conditions (1.2), i.e.

$\hat{x}\left(t, x_{0}, y_{0}\right)=x_{0}+\int_{\tau}^{t}\left[f\left(\tau, \beta(\tau, \alpha), \hat{x}\left(\tau, x_{0}, y_{0}\right), \mu, u\right)-\frac{1}{T} \int_{\tau}^{\tau+T} f(\tau, \beta(\tau, \alpha)\right.$,

$$
\left.\left.\hat{x}\left(\tau, x_{0}, y_{0}\right), \mu, u\right)\right] d \tau+\frac{t-\tau}{T}\left[e_{1} A_{2}^{-1}-\left(A_{1} A_{2}^{-1}+E\right) x_{0}\right]
$$

and

$\hat{y}\left(t, x_{0}, y_{0}\right)=y_{0}+\int_{\tau}^{t}\left[g\left(\tau, \beta(\tau, \alpha), \hat{y}\left(\tau, x_{0}, y_{0}\right), \omega, v\right)-\frac{1}{T} \int_{\tau}^{\tau+T} g(\tau, \beta(\tau, \alpha)\right.$,

$$
\left.\left.\hat{y}\left(\tau, x_{0}, y_{0}\right), \omega, v\right)\right] d \tau+\frac{t-\tau}{T}\left[e_{2} B_{2}^{-1}-\left(B_{1} B_{2}^{-1}+E\right) y_{0}\right]
$$

\footnotetext{
where

$\left.\mu=\int_{a}^{b} \beta(\tau, \alpha) \hat{x}\left(\tau, x_{0}, y_{0}\right) d \tau, \omega=\int_{c}^{d} \beta(\tau, \alpha)\right) \hat{y}\left(\tau, x_{0}, y_{0}\right) d \tau$

$u=\int_{-\infty}^{t} R(t, \tau)\left(\hat{x}\left(\tau, x_{0}, y_{0}\right)-\hat{y}\left(\tau, x_{0}, y_{0}\right)\right) d \tau$
} 
$v=\int_{-\infty}^{t} G(t, \tau)\left(\hat{x}\left(\tau, x_{0}, y_{0}\right)-\hat{y}\left(\tau, x_{0}, y_{0}\right)\right) d \tau$

Now, we shall prove that $\left(\begin{array}{c}x\left(t, x_{0}, y_{0}\right) \\ y\left(t, x_{0}, y_{0}\right)\end{array}\right)=\left(\begin{array}{c}\hat{x}\left(t, x_{0}, y_{0}\right) \\ \hat{y}\left(t, x_{0}, y_{0}\right)\end{array}\right)$ for all $t \in[\tau, \tau+T]$ and $x_{0} \in D_{\chi}, y_{0} \in D_{1 \chi}$. And to do this we need to prove the following inequality :-

$\left(\begin{array}{l}\left\|\hat{x}\left(t, x_{0}, y_{0}\right)-x_{m}\left(t, x_{0}, y_{0}\right)\right\| \\ \left\|\hat{y}\left(t, x_{0}, y_{0}\right)-y_{m}\left(t, x_{0}, y_{0}\right)\right\|\end{array}\right) \leq \Lambda^{m}(E-\Lambda)^{-1} \eta$

where

$M=\max _{t \in[\tau, \tau+T]}\left|f\left(t, \beta(\tau, \alpha), \hat{x}\left(t, x_{0}, y_{0}\right), \mu, u\right)\right|$

and

$N=\max _{t \in[\tau, \tau+T]}\left|g\left(t, \beta(\tau, \alpha), \hat{y}\left(t, x_{0}, y_{0}\right), \omega, v\right)\right|$

From (1.10), (1.11) and suppose $m=0$ in (2.13), we have

$\left\|\hat{x}\left(t, x_{0}, y_{0}\right)-x_{0}\right\|=\| x_{0}+\int_{\tau}^{t}\left[f\left(\tau, \beta(\tau, \alpha), \hat{x}\left(\tau, x_{0}, y_{0}\right), \int_{a}^{b} \beta(\tau, \alpha) \hat{x}\left(\tau, x_{0}, y_{0}\right) d \tau\right.\right.$,

$$
\begin{aligned}
& \left.\int_{-\infty}^{t} R(t, \tau)\left(\hat{x}\left(\tau, x_{0}, y_{0}\right)-\hat{y}\left(\tau, x_{0}, y_{0}\right)\right) d \tau\right)-\frac{1}{T} \int_{\tau}^{\tau+T} f\left(\tau, \beta(\tau, \alpha), \hat{x}\left(\tau, x_{0}, y_{0}\right),\right. \\
& \left.\left.\int_{a}^{b} \beta(\tau, \alpha) \hat{x}\left(\tau, x_{0}, y_{0}\right) d \tau, \int_{-\infty}^{t} R(t, \tau)\left(\hat{x}\left(\tau, x_{0}, y_{0}\right)-\hat{y}\left(\tau, x_{0}, y_{0}\right)\right) d \tau\right) d \tau\right] d \tau
\end{aligned}
$$

$+\frac{t-\tau}{T}\left[e_{1} A_{2}^{-1}-\left(A_{1} A_{2}^{-1}+E\right) x_{0}\right]-x_{0} \|$

Hence

$\left\|\hat{x}\left(t, x_{0}, y_{0}\right)-x_{0}\right\| \leq \alpha(t) M_{\beta} M+B_{1} \leq \frac{T}{2} M_{\beta} M+B_{1}$

By the same way, we get

$\left\|\hat{y}\left(t, x_{0}, y_{0}\right)-y_{0}\right\| \leq \alpha(t) N_{\beta} N+B_{2} \leq \frac{T}{2} N_{\beta} N+B_{2}$

Thus (2.17) is true for $m=0$, suppose that (2.18) and (2.19) are true for $m=p$, we have

$\left\|\hat{x}\left(t, x_{0}, y_{0}\right)-x_{p}\left(t, x_{0}, y_{0}\right)\right\| \leq \alpha(t) M_{\beta} C_{1}\left\|\hat{x}\left(t, x_{0}, y_{0}\right)-x_{p}\left(t, x_{0}, y_{0}\right)\right\|$

And similarly

$$
+\alpha(t) M_{\beta} C_{2}\left\|\hat{y}\left(t, x_{0}, y_{0}\right)-y_{p}\left(t, x_{0}, y_{0}\right)\right\|
$$

$\left\|\hat{y}\left(t, x_{0}, y_{0}\right)-y_{p}\left(t, x_{0}, y_{0}\right)\right\| \leq \alpha(t) N_{\gamma} C_{3}\left\|\hat{x}\left(t, x_{0}, y_{0}\right)-x_{p}\left(t, x_{0}, y_{0}\right)\right\|$

$$
+\alpha(t) N_{\gamma} C_{3}\left\|\hat{y}\left(t, x_{0}, y_{0}\right)-y_{p}\left(t, x_{0}, y_{0}\right)\right\|
$$

Rewrite the inequalities (1.34) and (1.35) in a vector form, we get

$\left(\begin{array}{c}\left\|\hat{x}\left(t, x_{0}, y_{0}\right)-x_{p}\left(t, x_{0}, y_{0}\right)\right\| \\ \left\|\hat{y}\left(t, x_{0}, y_{0}\right)-y_{p}\left(t, x_{0}, y_{0}\right)\right\|\end{array}\right) \leq \Lambda^{p}(E-\Lambda)^{-1} \eta$

Then, when $m=p+1$, we have

$\left\|\hat{x}\left(t, x_{0}, y_{0}\right)-x_{p+1}\left(t, x_{0}, y_{0}\right)\right\| \leq \alpha(t) M_{\beta} C_{1}\left\|\hat{x}\left(t, x_{0}, y_{0}\right)-x_{p}\left(t, x_{0}, y_{0}\right)\right\|$

$$
+\alpha(t) M_{\beta} C_{2}\left\|\hat{y}\left(t, x_{0}, y_{0}\right)-y_{p}\left(t, x_{0}, y_{0}\right)\right\|
$$

And similarly

$$
\begin{aligned}
\left\|\hat{y}\left(t, x_{0}, y_{0}\right)-y_{p+1}\left(t, x_{0}, y_{0}\right)\right\| & \leq \alpha(t) N_{\beta} C_{3}\left\|\hat{x}\left(t, x_{0}, y_{0}\right)-x_{p}\left(t, x_{0}, y_{0}\right)\right\| \\
& +\alpha(t) N_{\beta} C_{3}\left\|\hat{y}\left(t, x_{0}, y_{0}\right)-y_{p}\left(t, x_{0}, y_{0}\right)\right\|
\end{aligned}
$$


From (2.23) and (2.24) in vector form, we get

$\left(\begin{array}{l}\left\|\hat{x}\left(t, x_{0}, y_{0}\right)-x_{p+1}\left(t, x_{0}, y_{0}\right)\right\| \\ \left\|\hat{y}\left(t, x_{0}, y_{0}\right)-y_{p+1}\left(t, x_{0}, y_{0}\right)\right\|\end{array}\right) \leq \Lambda\left(\begin{array}{c}\left\|\hat{x}\left(t, x_{0}, y_{0}\right)-x_{p}\left(t, x_{0}, y_{0}\right)\right\| \\ \left\|\hat{y}\left(t, x_{0}, y_{0}\right)-y_{p}\left(t, x_{0}, y_{0}\right)\right\|\end{array}\right)$

Hence

$\leq \Lambda \Lambda^{p}(E-\Lambda)^{-1} \eta$

$\left(\begin{array}{l}\left\|\hat{x}\left(t, x_{0}, y_{0}\right)-x_{p+1}\left(t, x_{0}, y_{0}\right)\right\| \\ \left\|\hat{y}\left(t, x_{0}, y_{0}\right)-y_{p+1}\left(t, x_{0}, y_{0}\right)\right\|\end{array}\right) \leq \Lambda^{p+1}(E-\Lambda)^{-1} \eta$

Thus (2.17) is true in general.

So

$\lim _{m \rightarrow \infty}\left(\begin{array}{l}\left\|\hat{x}\left(t, x_{0}, y_{0}\right)-x_{m}\left(t, x_{0}, y_{0}\right)\right\| \\ \left\|\hat{y}\left(t, x_{0}, y_{0}\right)-y_{m}\left(t, x_{0}, y_{0}\right)\right\|\end{array}\right) \leq(E-\Lambda)^{-1} \eta \lim _{m \rightarrow \infty} \Lambda^{m}$.

Because $\lim _{m \rightarrow \infty} \Lambda^{m}=0$, and therefore

$\lim _{m \rightarrow \infty}\left(\begin{array}{l}\left\|\hat{x}\left(t, x_{0}, y_{0}\right)-x_{m}\left(t, x_{0}, y_{0}\right)\right\| \\ \left\|\hat{y}\left(t, x_{0}, y_{0}\right)-y_{m}\left(t, x_{0}, y_{0}\right)\right\|\end{array}\right)=0$

And hence

$\left(\begin{array}{l}\hat{x}\left(t, x_{0}, y_{0}\right) \\ \hat{y}\left(t, x_{0}, y_{0}\right)\end{array}\right)=\lim _{m \rightarrow \infty}\left(\begin{array}{l}x_{m}\left(t, x_{0}, y_{0}\right) \\ y_{m}\left(t, x_{0}, y_{0}\right)\end{array}\right)=\left(\begin{array}{l}x\left(t, x_{0}, y_{0}\right) \\ y\left(t, x_{0}, y_{0}\right)\end{array}\right)$

and this proves that the solution $\left(\begin{array}{c}x\left(t, x_{0}, y_{0}\right) \\ y\left(t, x_{0}, y_{0}\right)\end{array}\right)$ is a unique on the domain (2.15).

\section{EXISTENCE SOLUTION OF (1.1) WITH BOUNDARY CONDITION (1.2).}

The problem of the existence solution for the system (1.1) with boundary condition (1.2) is uniquely connected with the existence of zeros of the vector function :-

$\left(\begin{array}{l}\Delta_{1}^{*}\left(0, x_{0}, y_{0}\right) \\ \Delta_{2}^{*}\left(0, x_{0}, y_{0}\right)\end{array}\right)=\left(\begin{array}{l}\left.\frac{1}{T} \int_{\tau}^{\tau+T} f\left(\tau, \gamma \beta(\tau, \alpha), x\left(\tau, x_{0}, y_{0}\right), \mu, u\right) d \tau+\frac{1}{T}\left[A_{1} A_{2}^{-1}+E\right) x_{0}-e_{1} A_{2}^{-1}\right] \\ \left.\frac{1}{T} \int_{\tau}^{\tau+T} g\left(\tau, \beta(\tau, \alpha), y\left(\tau, x_{0}, y_{0}\right), \omega, v\right) d \tau+\frac{1}{T}\left[B_{1} B_{2}^{-1}+E\right) y_{0}-e_{2} B_{2}^{-1}\right]\end{array}\right)$

the vector function $\left(\begin{array}{c}\Delta_{1}^{*}\left(0, x_{0}, y_{0}\right) \\ \Delta_{2}^{*}\left(0, x_{0}, y_{0}\right)\end{array}\right)$ is approximately determined by the following :-

$\left(\begin{array}{c}\Delta_{1 m}^{*}\left(0, x_{0}, y_{0}\right) \\ \Delta_{2 m}^{*}\left(0, x_{0}, y_{0}\right)\end{array}\right)=\left(\begin{array}{l}\left.\frac{1}{T} \int_{\tau}^{\tau+T} f\left(\tau, \beta(\tau, \alpha), x_{m}\left(\tau, x_{0}, y_{0}\right), \mu_{m}, u_{m}\right) d \tau+\frac{1}{T}\left[A_{1} A_{2}^{-1}+E\right) x_{0}-e_{1} A_{2}^{-1}\right] \\ \left.\frac{1}{T} \int_{\tau}^{\tau+T} g\left(\tau, \beta(\tau, \alpha), y_{m}\left(\tau, x_{0}, y_{0}\right), \omega_{m}, v_{m}\right) d \tau+\frac{1}{T}\left[B_{1} B_{2}^{-1}+E\right) y_{0}-e_{2} B_{2}^{-1}\right]\end{array}\right)$

Theorem 3.1: If the hypotheses and all conditions of the theorem (3.1) are given then the following inequality are satisfy :-

$\left(\begin{array}{l}\left\|\Delta_{1}^{*}\left(0, x_{0}, y_{0}\right)-\Delta_{1 m}^{*}\left(0, x_{0}, y_{0}\right)\right\| \\ \left\|\Delta_{2}^{*}\left(0, x_{0}, y_{0}\right)-\Delta_{2 m}^{*}\left(0, x_{0}, y_{0}\right)\right\|\end{array}\right) \leq\left(\begin{array}{l}\left\langle\left(\begin{array}{l}d_{1} \\ d_{2}\end{array}\right), \Lambda^{m}(E-\Lambda)^{-1} \eta\right\rangle \\ \left\langle\left(\begin{array}{l}d_{3} \\ d_{4}\end{array}\right), \Lambda^{m}(E-\Lambda)^{-1} \eta\right\rangle\end{array}\right)$

where

$d_{1}=M_{\beta} C_{1}, d_{2}=M_{\beta} C_{2}, d_{3}=N_{\beta} C_{3}, d_{4}=N_{\beta} C_{4}$.

Proof . From the equations (3.1) and (3.2), we have 
$\left\|\Delta_{1}^{*}\left(0, x_{0}, y_{0}\right)-\Delta_{1 m}^{*}\left(0, x_{0}, y_{0}\right)\right\| \leq\left\langle\left(\begin{array}{l}d_{1} \\ d_{2}\end{array}\right), \Lambda^{m}(E-\Lambda)^{-1} \eta\right\rangle$

and

$\left\|\Delta_{2}^{*}\left(0, x_{0}, y_{0}\right)-\Delta_{2 m}^{*}\left(0, x_{0}, y_{0}\right)\right\| \leq\left\langle\left(\begin{array}{l}d_{3} \\ d_{4}\end{array}\right), \Lambda^{m}(E-\Lambda)^{-1} \eta\right\rangle$

From (3.4) and (3.5), we get (3.3).

Now, we prove the following theorem taking into account that the inequality (3.3) will be satisfy for all $m \geq 0, x_{0} \in D_{\chi}$, $y_{0} \in D_{1 \chi}$.

Theorem 3.2: Let the vector function $f(t, \gamma(t, \alpha), x, \mu, u)$ and $g(t, \gamma(t, \alpha), y, \omega, v)$ of the system (1.1) with boundary condition (1.2) are defined on the interval $[a, b]$ and $[c, d]$ in $R^{1}$. Assume that for any integer $m \geq 0$, the sequence of function (3.2) satisfies the function inequalities :-

$$
\left.\begin{array}{r}
\min _{x_{0} \in J_{1}, y_{0} \in J_{2}} \Delta_{1 m}^{*}\left(0, x_{0}, y_{0}\right) \leq-\left\langle\left(\begin{array}{l}
d_{1} \\
d_{2}
\end{array}\right), \Lambda^{m}(E-\Lambda)^{-1} \eta\right\rangle \\
\max _{x_{0} \in J_{1}, y_{0} \in J_{2}} \Delta_{1 m}^{*}\left(0, x_{0}, y_{0}\right) \geq\left\langle\left(\begin{array}{l}
d_{1} \\
d_{2}
\end{array}\right), \Lambda^{m}(E-\Lambda)^{-1} \eta\right\rangle
\end{array}\right\}
$$

where $x_{0} \in J_{1}=\left[a+\frac{T}{2} M_{\beta} M+B_{1}, b-\frac{T}{2} M_{\beta} M-B_{1}\right]$

and

$y_{0} \in J_{2}=\left[c+\frac{T}{2} N_{\beta} N+B_{1}, d-\frac{T}{2} N_{\beta} N-B_{2}\right]$.

Then (1.1) with boundary condition (1.2) has a periodic solution

$\left(\begin{array}{l}x \\ y\end{array}\right)=\left(\begin{array}{c}x\left(t, x_{0}, y_{0}\right) \\ y\left(t, x_{0}, y_{0}\right)\end{array}\right)$

for $x_{0} \in\left[a+J_{1}, b-J_{1}\right]$ and $y_{0} \in\left[c+J_{2}, d-J_{2}\right]$.

Proof . Let $x_{1}, x_{2}$ and $y_{1}, y_{2}$ be any points belonging on the intervals $I_{1}$ and $I_{2}$ respectively, such that

$\Delta_{1 m}^{*}\left(0, x_{1}, y_{1}\right)=\min _{x_{0} \in I_{1}, y_{0} \in I_{2}} \Delta_{1 m}^{*}\left(0, x_{0}, y_{0}\right)$

$\left.\Delta_{1 m}^{*}\left(0, x_{2}, y_{2}\right)=\max _{x_{0} \in I_{1}, y_{0} \in I_{2}} \Delta_{1 m}^{*}\left(0, x_{0}, y_{0}\right)\right\}$

$\Delta_{2 m}^{*}\left(0, x_{1}, y_{1}\right)=\min _{x_{0} \in I_{1}, y_{0} \in I_{2}} \Delta_{2 m}^{*}\left(0, x_{0}, y_{0}\right)$

$\left.\Delta_{2 m}^{*}\left(0, x_{2}, y_{2}\right)=\max _{x_{0} \in I_{1}, y_{0} \in I_{2}} \Delta_{2 m}^{*}\left(0, x_{0}, y_{0}\right)\right\}$

By using the inequalities (3.4), (3.5), (3.6), (3.7) and (3.8), we obtains

$\left.\left.\begin{array}{l}\Delta_{1}^{*}\left(0, x_{1}, y_{1}\right)=\Delta_{1 m}^{*}\left(0, x_{1}, y_{1}\right)+\left(\Delta_{1}^{*}\left(0, x_{1}, y_{1}\right)-\Delta_{1 m}^{*}\left(0, x_{1}, y_{1}\right)\right)<0 \\ \Delta_{1}^{*}\left(0, x_{2}, y_{2}\right)=\Delta_{1 m}^{*}\left(0, x_{2}, y_{2}\right)+\left(\Delta_{1}^{*}\left(0, x_{2}, y_{2}\right)-\Delta_{1 m}^{*}\left(0, x_{2}, y_{2}\right)\right)>0\end{array}\right\} \begin{array}{l}\Delta_{2}^{*}\left(0, x_{1}, y_{1}\right)=\Delta_{2 m}^{*}\left(0, x_{1}, y_{1}\right)+\left(\Delta_{2}^{*}\left(0, x_{1}, y_{1}\right)-\Delta_{2 m}^{*}\left(0, x_{1}, y_{1}\right)\right)<0 \\ \Delta_{2}^{*}\left(0, x_{1}, y_{1}\right)=\Delta_{2 m}^{*}\left(0, x_{2}, y_{2}\right)+\left(\Delta_{2}^{*}\left(0, x_{2}, y_{2}\right)-\Delta_{2 m}^{*}\left(0, x_{2}, y_{2}\right)\right)>0\end{array}\right\}$

and from the continuity of the functions $\Delta_{1}^{*}\left(0, x_{0}, y_{0}\right)$ and $\Delta_{2}^{*}\left(0, x_{0}, y_{0}\right)$ and the inequalities(3.10) and (3.11), then there exist and isolated points $x^{0} \in\left[x_{1}, x_{2}\right]$ and $y^{0} \in\left[y_{1}, y_{2}\right]$ such that $\Delta_{1}^{*}\left(0, x_{0}, y_{0}\right)=0$ and $\Delta_{2}^{*}\left(0, x_{0}, y_{0}\right)=0$. This means that (1.1) has a solution $\left(\begin{array}{l}x \\ y\end{array}\right)=\left(\begin{array}{l}x\left(t, x_{0}, y_{0}\right) \\ y\left(t, x_{0}, y_{0}\right)\end{array}\right)$.

\section{STABILITY OF SOLUTION OF (1.1) WITH BOUNDARY CONDITION (1.2).}

In this section, we study the stability theorem of a periodic solution for (1.1).

Theorem 4.1: Suppose that the function (3.1) be given. Then the following inequalities :

$$
\left(\begin{array}{l}
\left\|\Delta_{1}^{*}\left(0, x_{0}, y_{0}\right)\right\| \\
\left\|\Delta_{2}^{*}\left(0, x_{0}, y_{0}\right)\right\|
\end{array}\right) \leq\left(\begin{array}{c}
M_{\beta} M+\frac{B_{1}}{T} \\
N_{\beta} N+\frac{B_{2}}{T}
\end{array}\right)
$$

and 
$\left(\begin{array}{l}\left\|\Delta_{1}^{*}\left(0, x_{0}^{1}, y_{0}^{1}\right)-\Delta_{1}^{*}\left(0, x_{0}^{2}, y_{0}^{2}\right)\right\| \\ \left\|\Delta_{2}^{*}\left(0, x_{0}^{1}, x_{0}^{1}\right)-\Delta_{2}^{*}\left(0, x_{0}^{2}, y_{0}^{2}\right)\right\|\end{array}\right) \leq\left(\begin{array}{cc}R_{1} & R_{2} \\ R_{3} & R_{4}\end{array}\right)\left(\begin{array}{l}\left\|x_{0}^{1}-x_{0}^{2}\right\| \\ \left\|y_{0}^{1}-y_{0}^{2}\right\|\end{array}\right)$

are holds for all $x_{0}, x_{0}^{1}, x_{0}^{2} \in D_{\chi}$ and $y_{0}, y_{0}^{1}, y_{0}^{2} \in D_{1 \chi}$, where

$B_{1}=\left\|\left(A_{1} A_{2}^{-1}+E\right) x_{0}-e_{1} A_{2}^{-1}\right\|, B_{2}=\left\|\left(B_{1} B_{2}^{-1}+E\right) y_{0}-e_{2} B_{2}^{-1}\right\|$,

$B_{3}=\left\|A_{1} A_{2}^{-1}+E\right\|, B_{4}=\left\|B_{1} B_{2}^{-1}+E\right\|, F_{1}=\left[\left(1-\frac{T}{2} M_{\beta} C_{1}\right)\left(1-\frac{T}{2} N_{\beta} C_{4}\right)\right]^{-1}$,

$F_{2}=\left(1-\frac{T^{2}}{4} N_{\beta} M_{\beta} C_{2} C_{3} F_{1}\right)^{-1}, w_{1}=F_{1} F_{2}\left(1-\frac{T}{2} N_{\beta} C_{4}\right)\left(E+B_{3}\right)$,

$w_{2}=\frac{T}{2} M_{\beta} C_{2} F F_{2}\left(E+B_{4}\right), w_{3}=\frac{T}{2} N_{\beta} C_{3} F_{1} F_{2}\left(E+B_{3}\right)$,

$w_{4}=F_{1}\left(1-\frac{T}{2} M_{\beta} C_{1}\right)\left(E+B_{4}\right)\left[1+\frac{T^{2}}{4} N_{\beta} M_{\beta} C_{2} C_{3} F_{1} F_{2}\right]$,

$R_{1}=M_{\beta}\left(C_{1} w_{1}+C_{2} w_{3}\right)+\frac{B_{3}}{T}, R_{2}=M_{\gamma}\left(C_{1} w_{2}+C_{2} w_{4}\right), R_{3}=N_{\beta}\left(C_{3} w_{1}+C_{4} w_{3}\right)$,

$R_{4}=N_{\beta}\left(C_{3} w_{2}+C_{4} w_{4}\right)+\frac{B_{4}}{T}$

Proof . From the equation (3.1), we get

$\left\|\Delta_{1}^{*}\left(0, x_{0}, y_{0}\right)\right\| \leq M_{\beta} M+\frac{B_{1}}{T}$

And

$\left\|\Delta_{2}^{*}\left(0, x_{0}, y_{0}\right)\right\| \leq N_{\beta} N+\frac{B_{2}}{T}$

From (4.3) and (4.4), we get (4.1).

Now, by using the function (3.1), we get

$\left\|\Delta_{1}^{*}\left(0, x_{0}^{1}, y_{0}^{1}\right)-\Delta_{1}^{*}\left(0, x_{0}^{2}, y_{0}^{2}\right)\right\| \leq M_{\beta} C_{1}\left\|x\left(t, x_{0}^{1}, y_{0}^{1}\right)-x\left(t, x_{0}^{2}, y_{0}^{2}\right)\right\|$

$$
+M_{\beta} C_{2}\left\|y\left(t, x_{0}^{1}, y_{0}^{1}\right)-y\left(t, x_{0}^{2}, y_{0}^{2}\right)\right\|+\frac{B_{3}}{T}\left\|x_{0}^{1}-x_{0}^{2}\right\|
$$

And

$\left\|\Delta_{2}^{*}\left(0, x_{0}^{1}, y_{0}^{1}\right)-\Delta_{2}^{*}\left(0, x_{0}^{2}, y_{0}^{2}\right)\right\| \leq N_{\beta} C_{3}\left\|x\left(t, x_{0}^{1}, y_{0}^{1}\right)-x\left(t, x_{0}^{2}, y_{0}^{2}\right)\right\|$

$$
+N_{\beta} C_{4}\left\|y\left(t, x_{0}^{1}, y_{0}^{1}\right)-y\left(t, x_{0}^{2}, y_{0}^{2}\right)\right\|+\frac{B_{4}}{T}\left\|y_{0}^{1}-y_{0}^{2}\right\|
$$

where $x\left(t, x_{0}^{1}, y_{0}^{1}\right), x\left(t, x_{0}^{2}, y_{0}^{2}\right), y\left(t, x_{0}^{1}, y_{0}^{1}\right)$ and $y\left(t, x_{0}^{2}, y_{0}^{2}\right)$ are solutions of the integral equations :-

$$
\begin{aligned}
x\left(t, x_{0}^{k}, y_{0}^{k}\right)=x_{0}^{k}+\int_{\tau}^{t}\left[f\left(\tau, \beta(\tau, \alpha), x\left(\tau, x_{0}^{k}, y_{0}^{k}\right), \mu, u\right)-\frac{1}{T} \int_{\tau}^{\tau+T} f(\tau, \beta(\tau, \alpha)\right. \\
\left.\left., x\left(\tau, x_{0}^{k}, y_{0}^{k}\right), \mu, u\right)\right] d \tau+\frac{t-\tau}{T}\left[e_{1} A_{2}^{-1}-\left(A_{1} A_{2}^{-1}+E\right) x_{0}^{k}\right]
\end{aligned}
$$

$y\left(t, x_{0}^{k}, y_{0}^{k}\right)=y_{0}^{k}+\int_{\tau}^{t}\left[g\left(\tau, \beta(\tau, \alpha), y\left(\tau, x_{0}^{k}, y_{0}^{k}\right), \omega, v\right)-\frac{1}{T} \int_{\tau}^{\tau+T} g(\tau, \beta(\tau, \alpha)\right.$

$$
\left.\left., y\left(\tau, x_{0}^{k}, y_{0}^{k}\right), \omega, v\right)\right] d \tau+\frac{t-\tau}{T}\left[e_{2} B_{2}^{-1}-\left(B_{1} B_{2}^{-1}+E\right) y_{0}^{k}\right]
$$

with

$x\left(t, x_{0}^{k}, y_{0}^{k}\right)=x_{0}^{k}, y\left(t, x_{0}^{k}, y_{0}^{k}\right)=y_{0}^{k}$, where $k=1,2$.

Now, use the equation (4.7), as follows

$\left\|x\left(t, x_{0}^{1}, y_{0}^{1}\right)-x\left(t, x_{0}^{2}, y_{0}^{2}\right)\right\| \leq \frac{T}{2} M_{\beta} C_{1}\left\|x\left(t, x_{0}^{1}, y_{0}^{1}\right)-x\left(t, x_{0}^{2}, y_{0}^{2} y_{0}\right)\right\|$

$$
+\frac{T}{2} M_{\beta} C_{2}\left\|y\left(t, x_{0}^{1}, y_{0}^{1}\right)-y\left(t, x_{0}^{2}, y_{0}^{2} y_{0}\right)\right\|+\left(E+B_{3}\right)\left\|x_{0}^{1}-x_{0}^{2}\right\|
$$

then we can write

$\left\|x\left(t, x_{0}^{1}, y_{0}^{1}\right)-x\left(t, x_{0}^{2}, y_{0}^{2}\right)\right\| \leq\left(1-\frac{T}{2} M_{\beta} C_{1}\right)^{-1}\left(E+B_{3}\right)\left\|x_{0}^{1}-x_{0}^{2}\right\|$

Also, use the equation (4.8), we get

$$
+\frac{T}{2} M_{\beta} C_{2}\left(1-\frac{T}{2} M_{\gamma} C_{1}\right)^{-1}\left\|y\left(t, x_{0}^{1}, y_{0}^{1}\right)-y\left(t, x_{0}^{2}, y_{0}^{2} y_{0}\right)\right\|
$$


$\left\|y\left(t, x_{0}^{1}, y_{0}^{1}\right)-y\left(t, x_{0}^{2}, y_{0}^{2}\right)\right\| \leq \frac{T}{2} N_{\beta} C_{3}\left\|x\left(t, x_{0}^{1}, y_{0}^{1}\right)-x\left(t, x_{0}^{2}, y_{0}^{2} y_{0}\right)\right\|$

then we write this equation as follows

$$
+\frac{T}{2} N_{\beta} C_{4}\left\|y\left(t, x_{0}^{1}, y_{0}^{1}\right)-y\left(t, x_{0}^{2}, y_{0}^{2} y_{0}\right)\right\|+\left(E+B_{4}\right)\left\|y_{0}^{1}-y_{0}^{2}\right\|
$$

$$
\begin{aligned}
\left\|y\left(t, x_{0}^{1}, y_{0}^{1}\right)-y\left(t, x_{0}^{2}, y_{0}^{2}\right)\right\| \leq\left(1-\frac{T}{2} N_{\gamma} C_{4}\right)^{-1}(E+ & \left.B_{4}\right)\left\|y_{0}^{1}-y_{0}^{2}\right\| \\
& +\frac{T}{2} M_{\beta} C_{3}\left(1-\frac{T}{2} N_{\gamma} C_{4}\right)^{-1}\left\|x\left(t, x_{0}^{1}, y_{0}^{1}\right)-x\left(t, x_{0}^{2}, y_{0}^{2} y_{0}\right)\right\|
\end{aligned}
$$

By substituting inequality (4.12) in (4.10), we get

$$
\begin{aligned}
\left\|x\left(t, x_{0}^{1}, y_{0}^{1}\right)-x\left(t, x_{0}^{2}, y_{0}^{2}\right)\right\| \leq F_{1} F_{2}\left(1-\frac{T}{2} N_{\gamma} C_{4}\right)\left(E+B_{3}\right)\left\|x_{0}^{1}-x_{0}^{2}\right\| & \\
& +\frac{T}{2} M_{\beta} C_{2} F_{1} F_{2}\left(E+B_{4}\right)\left\|y_{0}^{1}-y_{0}^{2}\right\|
\end{aligned}
$$

Also, substituting the inequality (4.12) in (4.14), we find that

$$
\begin{array}{r}
\left\|y\left(t, x_{0}^{1}, y_{0}^{1}\right)-y\left(t, x_{0}^{2}, y_{0}^{2}\right)\right\| \leq \frac{T}{2} N_{\beta} C_{3} F_{1} F_{2}\left(E+B_{3}\right)\left\|x_{0}^{1}-x_{0}^{2}\right\|+F_{1}\left(1-\frac{T}{2} M_{\gamma} C_{1}\right) \\
\left(E+B_{4}\right)\left[1+\frac{T^{2}}{4} N_{\beta} M_{\beta} C_{2} C_{3} F_{1} F_{2}\right]\left\|y_{0}^{1}-y_{0}^{2}\right\|
\end{array}
$$

Finally, we substitute the inequalities (4.13) and (4.14) in (4.5), we get (4.2)and substitute the inequalities (4.13) and (4.14) in (4.6), we get (4.2).

Theorem 4.2: Let the system (1.1) with boundary conditions (1.2) be defined in the domain (1.3). Suppose that $G_{1}$ and $G_{2}$ be closed and bounded domain subset of domain $D_{\chi}$ and $D_{1 \chi}$. Then, $G_{1}$ and $G_{2}$ have points at which the $\Delta-$ constant is zero, then for any point $x_{0} \in D_{\chi}$ and $y_{0} \in D_{1 \chi}$, the following inequality holds :-

$$
\left(\begin{array}{l}
\left\|\Delta_{1 m}^{*}\left(0, x_{0}, y_{0}\right)\right\| \\
\left\|\Delta_{2 m}^{*}\left(0, x_{0}, y_{0}\right)\right\|
\end{array}\right) \leq\left(\begin{array}{c}
\left\langle\left(\begin{array}{l}
d_{1} \\
d_{2}
\end{array}\right), \Lambda^{m}(E-\Lambda)^{-1} \eta\right\rangle \\
\left\langle\left(\begin{array}{l}
d_{3} \\
d_{4}
\end{array}\right), \Lambda^{m}(E-\Lambda)^{-1} \eta\right\rangle
\end{array}\right)+\left(\begin{array}{c}
M_{\beta} M+\frac{B_{1}}{T} \\
N_{\beta} N+\frac{B_{2}}{T}
\end{array}\right)
$$

for all $m \geq 0$ and $x_{0} \in D_{\chi}, y_{0} \in D_{1 \chi}$.

Proof. By using the inequality (4.1), we get

$\left\|\Delta_{1}^{*}\left(0, x_{0}, y_{0}\right)\right\| \leq M_{\beta} M+\frac{B_{1}}{T}$

and

$\left\|\Delta_{2}^{*}\left(0, x_{0}, y_{0}\right)\right\| \leq N_{\beta} N+\frac{B_{2}}{T}$

Also, from (3.1), we have

$\left\|\Delta_{1 m}^{*}\left(0, x_{0}, y_{0}\right)\right\|=\left\|\Delta_{1 m}^{*}\left(0, x_{0}, y_{0}\right)-\Delta_{1}^{*}\left(0, x_{0}, y_{0}\right)+\Delta_{1}^{*}\left(0, x_{0}, y_{0}\right)\right\|$

$\leq\left\|\Delta_{1 m}^{*}\left(0, x_{0}, y_{0}\right)-\Delta_{1}^{*}\left(0, x_{0}, y_{0}\right)\right\|+\left\|\Delta_{1}^{*}\left(0, x_{0}, y_{0}\right)\right\|$

$$
\leq\left\langle\left(\begin{array}{l}
d_{1} \\
d_{2}
\end{array}\right), \Lambda^{m}(E-\Lambda)^{-1} \eta\right\rangle+\left(M_{\beta} M+\frac{B_{1}}{T}\right)
$$

and

$\left\|\Delta_{2 m}^{*}\left(0, x_{0}, y_{0}\right)\right\|=\left\|\Delta_{2 m}^{*}\left(0, x_{0}, y_{0}\right)-\Delta_{2}^{*}\left(0, x_{0}, y_{0}\right)+\Delta_{2}^{*}\left(0, x_{0}, y_{0}\right)\right\|$

$$
\leq\left\|\Delta_{2 m}^{*}\left(0, x_{0}, y_{0}\right)-\Delta_{2}^{*}\left(0, x_{0}, y_{0}\right)\right\|+\left\|\Delta_{2}^{*}\left(0, x_{0}, y_{0}\right)\right\|
$$

$$
\leq\left\langle\left(\begin{array}{l}
d_{3} \\
d_{4}
\end{array}\right), \Lambda^{m}(E-\Lambda)^{-1} \eta\right\rangle+\left(N_{\beta} N+\frac{B_{2}}{T}\right)
$$

Rewrite the inequalities (4.16) and (4.17) in a vector form, we get (4.15).

\section{REFERENCES}

[1] Ali, A. R., periodic solutions for some classes of non-linear systems of integro-differential equations, M.Sc. Thesis, college of Scince, Duhok University, Duhok, (2010).

[2] Aziz, M. A., periodic solutions for some systems of non-linear ordinary differential equations, M.Sc. Thesis, college of Education, University of Mosul, (2006).

[3] Benaicha, S., periodic boundary value problems for second order ordinary differential equations, University of Oran, Es-senia, Algeria, Applied Mathematical Scinces, Vol. 3, No. 6, pp. 267-276, (2009). http://www.m-hikari.com/ams/ams-password-2009/ams-password5-82009/benaichaAMS5-8-2009.pdf

[4] Bttelli, F. and Feckan, M., Handbook of Differential Equations Ordinary Differential Equations, first edition, Amsterdam, Vol. 4, pp.441-490, (2008).

file:///C:/Users/Home1/Downloads/[Flaviano_Battelli,_Michal_Feckan]_Handbook_of_Dif(z-lib.org).pdf

[5] Butris, R. N., existence of solution for systems of second order differential equations with boundary conditions, J. of Education and since, Mosul, Iraq, Vol. 18, (1994). 
[6] Buttris, R. N., and Jameel, Gh. Sh., periodic solutions for nonlinear system of differential equations of third order with boundary conditions, J. Edu. \& Sci., Vol. 26, No. 1, (2013). https://www.iasj.net/iasj?func=fulltext\&aId=89560

[7] Butris, R. N. and Shammo, S. Kh., periodic solutions for non-linear systems of differential equations with boundary conditions, Iraq, Vol. 5(3), (2014). http://www.ijma.info/index.php/ijma/article/view/2783/1704

[8] Butris, R. N. and Taher, R. F., periodic solution of integro-differential equations depended on special function with singular kernels, international Journal of Mechanical Engineering and Technology (IJMET), Vol. 10, Issue 04, pp. 786-800, Article ID:IJMET_10_04_078. http://www.iaeme.com/MasterAdmin/uploadfolder/IJMET_10_04_078/IJMET_10_04_078.pdf

[9] James, R. W., periodic solution of ordinary differential equations with boundary nonlinearities, J. of the Julius Schauder Center Vol. 19, pp. 257282, (2002). https://projecteuclid.org/download/pdf_1/euclid.tmna/1470138764

[10] Jankowski, T., Numerical-analytic method for implicit differential equations, Mathematical Notes, Miskole, Vol. 2, No. 2, pp. 137-144, (2001). file://C:/Users/Home1/Downloads/1988/jankowski\%202001.pdf

[11] Kiguradze and Puza, M., On periodic solutions of non-linear functional differential equations, Georgian Math. J. Vol. 6, No. 1, pp. 45-64, (1999). https://link.springer.com/article/10.1023/A:1022978326156\#citeas

[12] Mitropolsky, Yu. A. and Martynyuk, D. I., For periodic solutions for the oscillations system with retarded argument, Kiev, Ukraine, General School, (1979).

[13] Narjanov, O. D., On a periodic solution for integro-differential equation, Math. J., Kiev, Ukraine, Tom. 2, (1977).

[14] Naima, D. M., periodic solution of non-autonomous second-order differential equations and boundary value problem, India, Issue. 6, (1999).

[15] Perestyuk, N. A. and Martynyuk, D. I., periodic solutions of a certain class systems of differential equations, Math. J. Univ. of Kiev, Kiev, Ukraine, Tom. 3, pp. 146-156, (1976).

[16] Putertnka, T. V., The convergent method for nonlinear system of differential equation with boundary integral conditions, Nonlinear problems in the thory of differential equations J. Akad Nauk, Ukraine, Tom. 9, pp. 66-73, (1991).

[17] Rafeq, A. Sh., Periodic solutions for non-linear systems of differential equations with matrices, J. Duhok Univ. Vol. 14, No. 1, PP. 57-74, (2013).

[18] Rafeq, A. Sh., Periodic solutions for some of non-linear systems of integro -differential equations, M. Sc. Thesis, college of Science, Duhok University, Duhok, (2009).

[19] Ronto, A., Ronto, M. and Shchobak, N. ,On numerical-analytic methods techniques for boundary value problem, Acta Electrotechnica et Informatica, Vol. 12, No. 3, pp. 67-72, DOI: 10.2478/v10198-012--0035-1, (2012).

[20] Ronto, A. and Ronto, M., On the investigation of some boundary value problems with non- linear conditions, Mathematical Notes, Miskolc, Vol. 1, No. 1, pp. 43-55, (2000).

[21] Samoilenko, A.M. and Ronton, N. I., A numerical analytic methods for investigations of periodic solutions, Kiev, Ukraine, (1976).

[22] Samoilenko, A. M. and Ronto, N. I., Numerical- analytic methods for investigating solutions of boundary value problem, Kiev, Ukraine, (1985).

[23] Shslapk, Yu. D.Periodic solutions of first-order nonlinear differential equations unsolvable for derivative, Math. J. Ukraine, Kiev, Ukraine, Tom. 5, pp. 850-854, (1980).

[24] Zavizion, G.V., periodic solutions of integro-differential equations, J. of Diff. Equations, Vol. 45, No. 2, pp. 240-248, (2009).

[25] Zafer, A., Differential Equations and Their Applications, Second Edition, New Delhi, (2010). https://www.abebooks.com/products/isbn/9788120325234?cm_sp=rec-_-vhr_1-_-plp\&reftag=vhr_125. 